\title{
PV power forecasting using different artificial neural networks strategies
}

\author{
I.Sansa $^{1}$, S.Missaoui ${ }^{2}$, Z.Boussada ${ }^{3}$, N. Mrabet Bellaaj ${ }^{4}$ \\ University of Tunis El Manar \\ LSE-ENIT-LR11ES1 \\ Tunis, Tunisia \\ 1sansa.ines@yahoo.com, \\ ${ }^{2}$ missaouisihem@yahoo.com \\ ${ }^{3}$ zina.b.88@hotmail.fr, \\ ${ }^{4}$ najiba_bm@yahoo.fr
}

\begin{abstract}
The integration of photovoltaic (PV), intermittent and uncontrollable power, into the electrical grid has become one of the major challenges for power system operators. Therefore the $\mathrm{PV}$ power forecasting can be beneficial in system planning and balancing energies. In this paper the PV power forecasting of a real generator [1] is presented. Different Artificial Neural Networks (ANN) strategies are used to forecast the $P V$ power from meteorological variables, the radiation and the temperature. Simulation results correspond to each ANN strategy are presented, discussed and compared. The Nonlinear Auto Regressive models with eXogenous input (NARX model) is the dynamic ANN chosen to use in this work. Its performances have proved in the different time frame PV power forecasting. The impact of type season's on $\mathrm{PV}$ power forecasting performances is also presented in the second part of this paper.
\end{abstract}

Keywords-PV power forecasting; ANN; NARX model; model identification.

\section{INTRODUCTION}

Following the economic growth and the high consumption of energy in all the countries of the world, there has been in the last decade a dramatically increment on the use of renewable energy such as the PV energy. Despite the cleanness and the reliability of this type of energy, it has the problem of intermittence. Through photovoltaic panel, solar radiation is converted into electricity without combustion creating clean and abundant energy. The PV power was firstly used to supply devices that it is difficult to access it, it was the best solution for the tags in sea and in the isolated equipment. Thanks to its low cost and its simple installation the use of PV power increases rapidly. Today this source of energy is used in industries in a large part and it is the most appropriate in the production of electricity from renewable sources for habitat [2], [3]. Moreover to benefit ideally this power and to avoid its intermittence problem, it is so important to forecast the quantity of energy that will be produced after a day, a month or even after a year. The renewable energy forecasting and particularly PV power forecasting was the interest of

\author{
Emad Mahmoud \\ Aswan Faculty of engineering \\ APEARC \\ Aswan, Egypt \\ elbakoury@apearc.aswu.edu.eg
}

many researches and several methods have been proposed [412]. In [4] for forecasting PV power, the author uses models based on mathematical equations of PV cells and sun position [4]. Numerical weather predictions were introduced as inputs to these models. This method was not efficient by reason of the large PV plants that make the mathematical approach tedious and this is can lead to errors in physical models of this PV system [5]. Linear time series models are used in [6] where PV power was predicted by adaptive type models. It was demonstrated that historical solar data represent the most important input for the short forecasting while the Numerical weather predictions are important for the long forecasting. The regression analysis is used in [7] to predict a PV power output of a $220 \mathrm{~kW}$ system. A PV simulator is used taking radiation on inclined surfaces as input and the PV power forecasted is computed from the I-U characteristic of PV cell. This method cannot be efficient if the PV power output change rapidly in a non linear manner. Some other researchers in literature are interested to forecast firstly the radiation and then convert it into PV power. Autoregressive Moving Average (ARMA) and Autoregressive Integrated Moving Average (ARIMA) models are reported in [8] and [9], in these works to convert the radiation to power, linear models are introduced. This cannot be in the real case because the conversion from radiation to power usually based on equations taking into account the outside air temperature and the array area. Static ANNs are used to estimate the maximum power output of PV module [10]. In [13] ANNs are used to predict solar radiation by climatological variables. The temperature, the relative humidity, the mean vapor pressure and other variables are introduced as inputs to predict the solar global radiation. In [13] physical and statistical methods are studied and used for forecasting PV power from weather prediction information. The root mean square errors (RMSE) over one month for these two methods are around $10 \%$ to $12 \%$. It is shown in this paper that the main origin of these errors comes from accuracy of weather prediction information. In this present paper the PV power will be forecasted using different ANN strategies and for different time frame. Our choice of ANN as tool to forecast the PV power is not random but because of its universal approximator propriety. This forecasting will be just 
from the radiation and the temperature correspond to a region located in the north of Barcelona. All these meteorological data are instantaneous values captured with a data capture module. Static ANN and one type of dynamic ANN called NARX model are applied in this work. Then, PV power forecasting results are compared. The present paper is organized as follows; in the section 2, a PV model of a panel and a whole generator is presented. This model is useful to give as an approximation of the production of the studied system. The identification of some parameters is also presented to have a useful model such as the number of panel and the coefficient temperature. In the section 3, static ANN structure and NARX model are presented. Forecasting results correspond to short, medium and long time frame forecasting are analyzed and compared. In the following section, just NARX model will be used and to improve more its performance, the type of season is added as input to the neural structure. Finally a conclusion and discussion of results will be presented.

\section{MODEL IDENTIFICATION FOR PV POWER FORECASTING}

PV power is the result of solar radiation converted into electricity through photovoltaic panels which are a group of solar cells associated in series and/or in parallel. To explain the photovoltaic effect different physical properties are required. To have in details the principle of photovoltaic effect, the different types of cells and the mode of conversion of solar into electrical energy, reader can refer to [15]. In this part different equations are presented to express PV power from weather parameters. Beginning with the following one written in (1)

$$
P_{p v}=\eta \cdot S \cdot G \cdot N
$$

Where $\eta$ is the cell conversion yield, $S$ represents the module area, $\mathrm{G}$ is the radiation and $\mathrm{N}$ is the number of PV module. The cell conversion $\eta$ can be expressed as follow:

$$
\eta=\eta_{r} \cdot\left(1-\lambda \cdot\left(T_{c}-T_{r e f}\right)\right.
$$

With $\eta_{\mathrm{r}}$ is the cell conversion under standard conditions, $\lambda$ is the temperature coefficient. $\mathrm{T}_{\mathrm{c}}$ and $\mathrm{T}_{\text {ref }}$ are respectively the cell temperature and the reference temperature $\left(\mathrm{T}_{\text {ref }}=25^{\circ} \mathrm{C}\right)$. Writing the cell conversion yield according to (2), (1) is developed as (3).

$$
P_{p v}=\eta_{r} \cdot\left(1-\lambda \cdot\left(T_{c}-T_{r e f}\right) \cdot S \cdot G \cdot N\right.
$$

The cell conversion yield under standard conditions is written in terms of the module area $\mathrm{S}$, the maximum power $\mathrm{P}_{\max }$, and the reference radiation $\mathrm{G}_{\mathrm{ref}}$ as presented in (4)

$$
\eta_{r}=\frac{P_{\max }}{S . G_{\text {ref }}}
$$

Finally the PV power can be written as (5),

$$
P_{p v}=\frac{P_{\max }}{G_{r e f}} \cdot G \cdot N \cdot\left(1-\lambda \cdot\left(T_{c}-T_{\text {ref }}\right)\right)
$$

Normally the PV power is computed according to (5) but not all parameters in this equation are available because our data base contains just the PV power, the radiation and the temperature. In this task we interest to identify the number of PV module $(\mathrm{N})$ and the coefficient temperature $(\lambda)$. Then the PV power will be forecasted using these parameters. Equations (6) to (11) are the development of (5) to make this equation as a linear form. We begin firstly with the normalization of $\mathrm{PV}$ power, the radiation and the temperature as presented in (6).

$$
P_{p v n}=\frac{1}{G_{r e f}} \cdot G_{n} \cdot N \cdot\left(1-\lambda \cdot\left(T_{c n}-T_{r e f}\right)\right)
$$

With $P_{p v n}, G_{n}$ and $T_{c n}$ are the normalized $P V$ power, radiation and temperature.

Assuming:

$$
a=\frac{1}{G_{r e f}}, b=\lambda, c=T_{r e f}
$$

$\mathrm{P}_{\mathrm{pvn}}$ is written as

$$
P_{p v n}=a \cdot G_{n} \cdot N \cdot\left(1-b \cdot\left(T_{c n}-c\right)\right)
$$

The development of (8) leads to (9)

$$
P_{p v n}=a \cdot G_{n} \cdot(1+b \cdot c) \cdot N-a \cdot b G_{n} \cdot T_{c} \cdot N
$$

Assuming:

$$
A=a .(1+b . c) \cdot N \quad \text { And } \quad B=-a \cdot b \cdot N
$$

Equation (9) can be written as follows:

$$
P_{p v n}=A \cdot\left(G_{n}\right)+B \cdot\left(G_{n} \cdot T_{c}\right)
$$

Following (11) it's possible to estimate values of A and B using static ANN composed just of one neuron. The inputs will be the radiation $\left(\mathrm{G}_{\mathrm{n}}\right)$ and the product of the radiation and the temperature $\left(\mathrm{G}_{\mathrm{n}}, \mathrm{T}_{\mathrm{c}}\right)$. The transfer function is linear. After learning, $\mathrm{A}$ and $\mathrm{B}$ will be estimated respectively to the weights values $W_{11}$ and $W_{21}$. The neural model for the PV power forecasting is presented in the fig.1. The learning performance is presented in the fig. 2. The mean square error of learning (MSEL) obtained is equal to $8.5055 .10^{-5}$. Thus $\mathrm{N}$ and $\lambda$ are identified as explained in (12), (13) and (14).

$$
P_{p v n}=W_{1 i}\left(G_{n}\right)+W_{2 i}\left(G_{n} \cdot T_{c}\right)
$$


$\mathbf{G}_{\text {n }}$

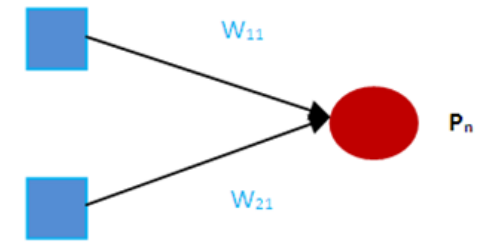

Fig.1. Neural model for PV power forecasting

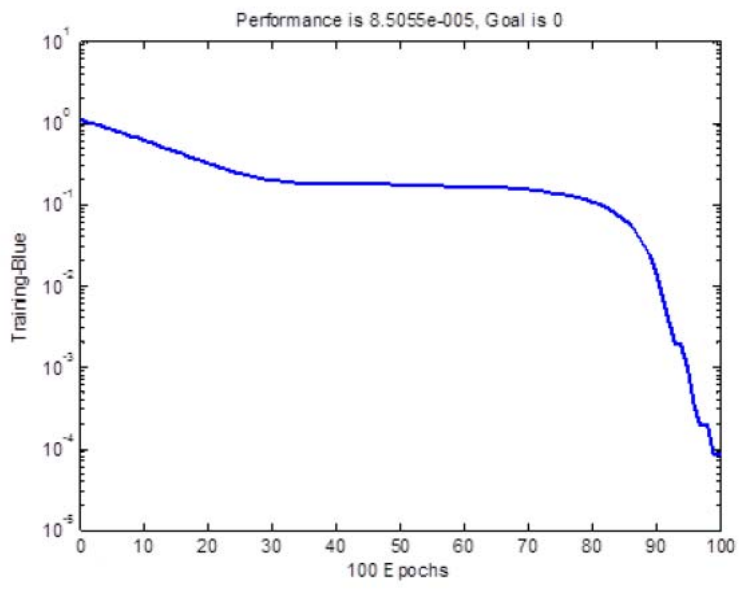

Fig.2. Learning performance

After learning, we found $\mathrm{W}_{11}$ and $\mathrm{W}_{21}$ respectively 1.3233 and -0.2927 .

$$
\left\{\begin{array}{l}
W_{11}=A=a \cdot(1+b \cdot c) \cdot N=1.3233 \\
W_{21}=B=-a \cdot b \cdot N=-0.2927
\end{array}\right.
$$

Finally:

$$
\left\{\begin{array}{l}
N=1223 \text { cells } \\
\lambda=0.0037
\end{array}\right.
$$

In theory the value of $\lambda$ is between 0.004 and 0.006 therefore its value obtained by identification is acceptable. Moreover it is interesting to check the forecasting performances of the model. In fact, all parameters of the PV module described by (5) are now well known. In the fig. 3 we present, for a chosen day, the real PV power, the forecasted one computed according (5) and the forecasting error.

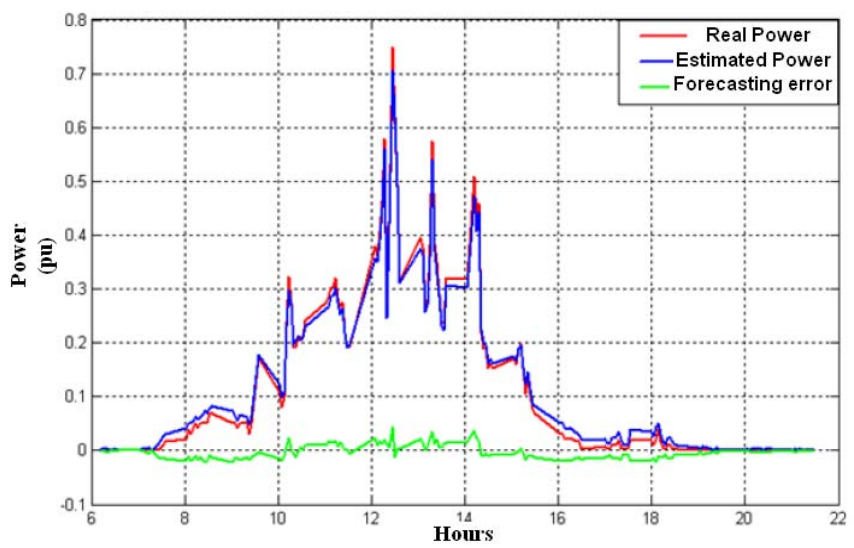

Fig.3. PV power forecasting using the identification model
As shown in the fig.3, the real and forecasted curves are so close, almost overlapped. The forecasting error is low, it reaches 0.005 . This is demonstrates the performance of the model identification to forecast the PV power.

\section{III.PV POWER FORECASTING USING ANN}

In this part we interest to forecast the PV power using static and dynamic ANN. This forecasting will be from the radiation and the temperature and for different time frame, the short, medium and long term. ANN represents a convenient method for forecasting. It is based on the theory of human brain to learn data and then to create relations between inputs and outputs. The graphic of Static ANN is a succession of interconnected neurons, and the information transmits from inputs to outputs without backward. Dynamic ANN has a form different to static ANN, it presents a delay related the outputs to the inputs. Fig. 4 and fig.5 represent respectively the neural structures of the static and a proposed dynamic ANN called NARX for the PV power forecasting.

\section{A. Long term forecasting}

In this task, the PV power is forecasted for 5 days. Applying cross validation method, the optimal structure obtained for the static ANN is composed of two neurons in the hidden layer. For NARX model the optimal structure contains three neurons in the hidden layer. Mean square errors of test (MSET) for static ANN and for NARX model are respectively $7.04310^{-4}$ and $5.776910^{-4}$. The fig.6 presents the forecasting errors in percent for the both types of ANN. Comparing the two curves we show that the error decreases from $17 \%$ using static ANN to $10 \%$ if we apply NARX. This is demonstrates the performance of NARX model on the PV power forecasting in a long term. To confirm these results, we superpose curves of real and PV power forecasted by the both types of ANN as presented in the fig.7.

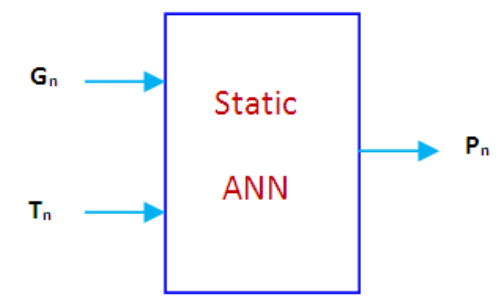

Fig.4.Static ANN structure for PV power forecasting

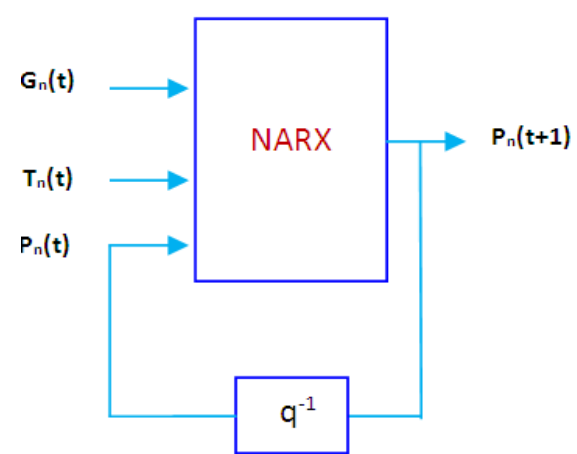

Fig.5. Dynamic ANN for PV power forecasting (NARX model) 


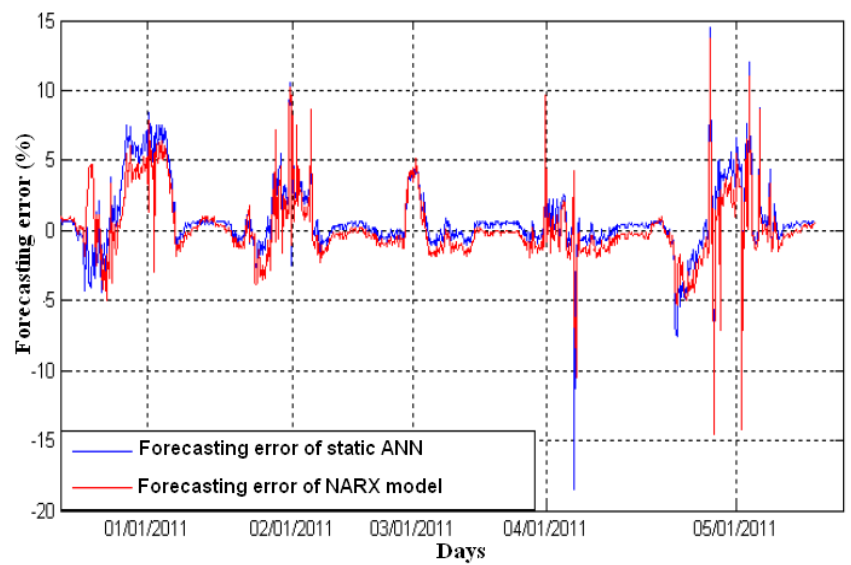

Fig.6. Forecasting errors for static ANN and NARX model in a long term

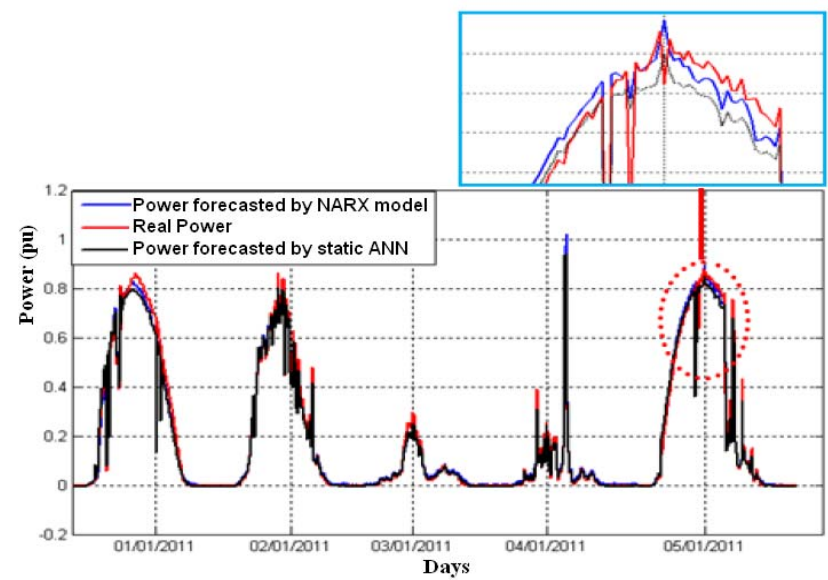

Fig.7. Long term PV power forecasting

\section{B. Medium term forecasting}

This category of forecasting is so important and it is over used especially for the dispatching of conventional power plants. In this part PV power is forecasted for a one day. The MSET using static ANN and NARX model are respectively 8.98.10 and $5.5 .10^{-5}$. The forecasting error decreases from $8 \%$ to $5 \%$ as presented in the fig. 8 . The performance of NARX model has also proved on the PV power forecasting in the medium term. As presented in the fig.9 the real PV power and this one forecasted by NARX model are almost overlapped.

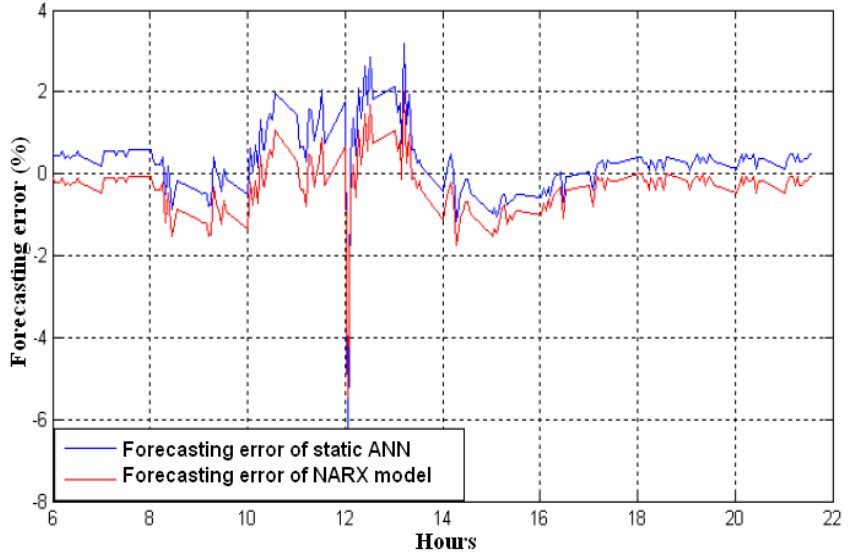

Fig.8. Forecasting errors for static ANN and NARX model in a medium term

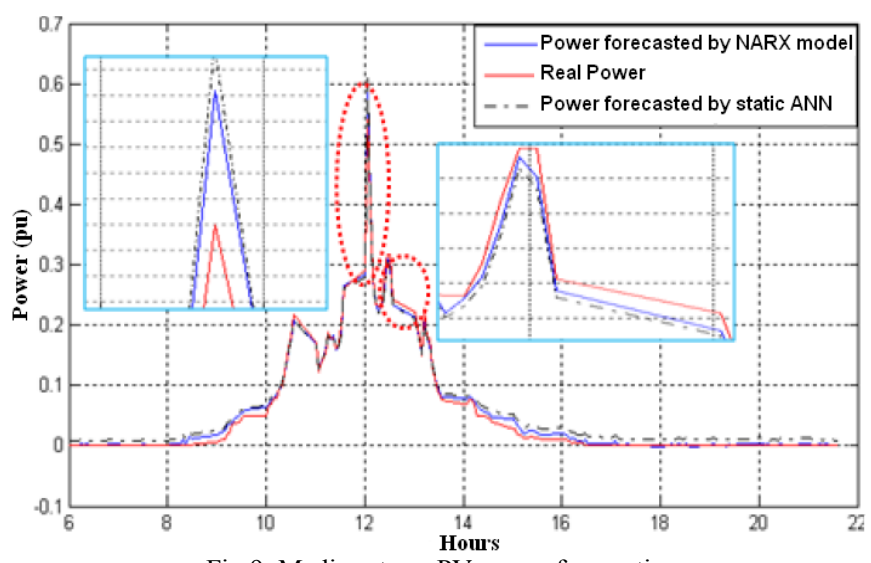

Fig.9. Medium term PV power forecasting

\section{Short term forecasting}

This category of forecasting is so interesting for the manager of network. In fact they forecast PV power in short term to supply the network energy provided by combustion engines when PV power cannot satisfy needs of consumers. As the case of previous types of forecasting, in this part we forecast PV power with static and dynamic ANN. Thus for this category of forecasting the performance of NARX is demonstrated. Indeed, the MSET obtained using static ANN reaches $5.404 .10^{-5}$ and $2.514 .10^{-5}$ after the use of NARX model. The forecasting error decreases slightly from $2.75 \%$ to $1.74 \%$ as presented in the fig. 10 . Results of forecasting in a short time of PV power are presented in the fig. 11.

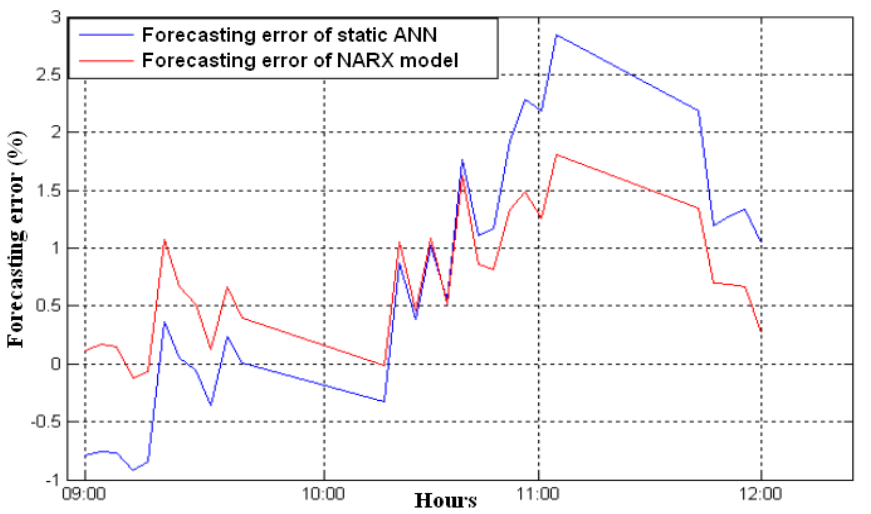

Fig.10. Forecasting errors for static ANN and NARX model in a short term

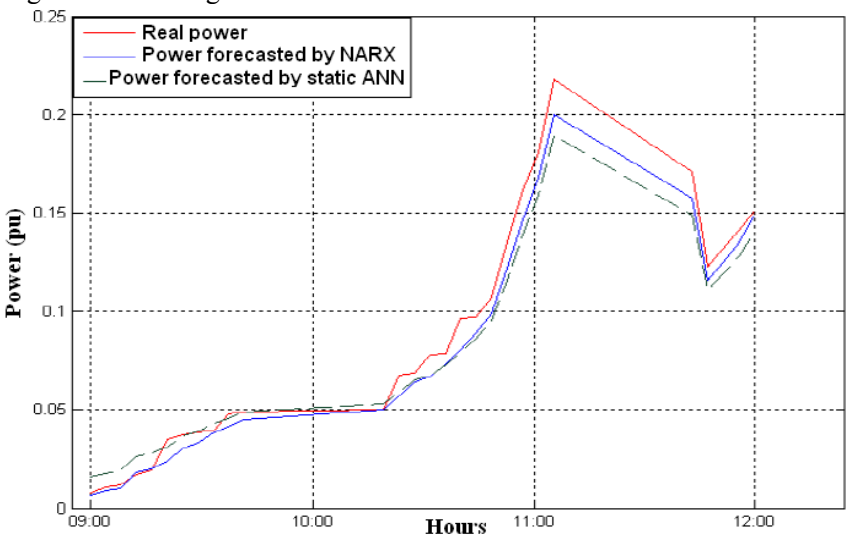

Fig.11. Short term PV power forecasting 


\section{IV.PV POWER FORECASTING USING AN IMPROVED STRUCTURE}

In this section we interest to improve the $\mathrm{PV}$ power forecasting adding another input called "class". Class reflects the type of season: "could" or "hot". This forecasting will be just with NARX model. The input "class" is not quantifiable, so it is assigned a coding for it. The code assigned to class "cold" is " 1 " and the code assigned to class "hot" is " 5 ". To ensure the importance of this input, we forecast the PV power for the different time frame. Then results will be compared with its obtained in the previous parts.

\section{A. Long term forecasting using the improved structure}

In this horizon and comparing to the previous results, the forecasting error decreases slightly. It reaches $6 \%$ as presented in the fig.12. Although the improvement is small but it has a significant effect on the dispatching of network especially on the great power. The introduce of the type of season as input to NARX model can improve the PV power forecasting on the long term as verified in the fig.13. In fact curves correspond to the real PV power and this one forecasted based to the improved structure are so close.

\section{B. Medium term forecasting using the improved structure}

We forecast the PV power using the new neural structure for a one day. The comparison between the both errors with the new and previous structures is presented in the fig. 14 .

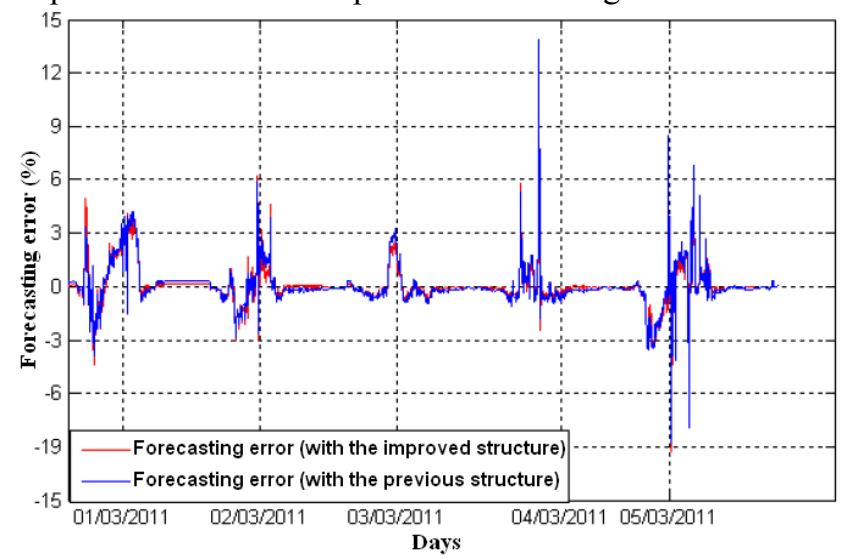

Fig.12. Forecasting error in the long term using the improved structure

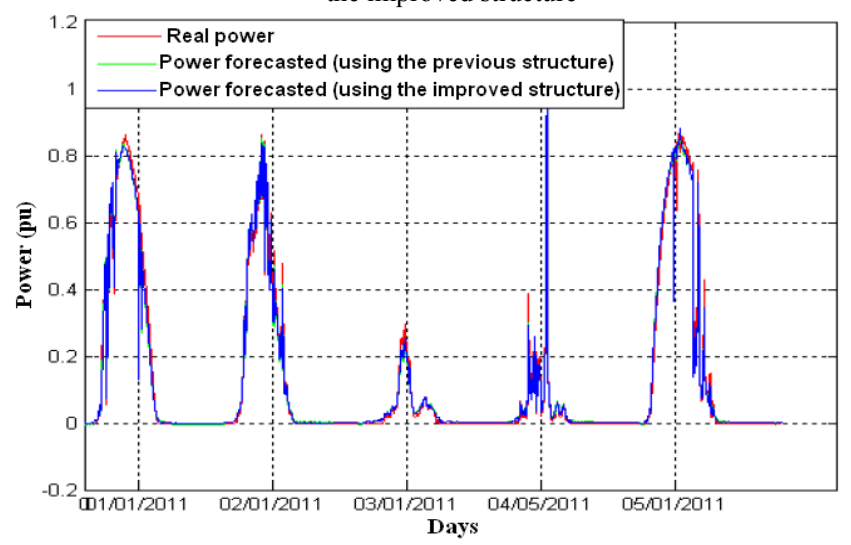

Fig.13. PV power forecasting on the long term using the improved structure
The maximum value of the forecasting error with the previous structure reaches $5 \%$ and with the new structure it doesn't exceed the $2 \%$. The performances of forecasting are improved also in the medium term as validated in the fig. 15 . The PV power forecasted by NARX model using the new structure is almost confused to the real power.

\section{Short term forecasting using the improved structure}

In this category the performances of forecasting is also verified when the type of season is taking into account. As presented in the fig. 16 the forecasting error is too low not exceed the $1.2 \%$. The impact of season is obvious on the short term PV power forecasting as shown in the fig. 17. In the table I we recapitulate the different results obtained in this work.

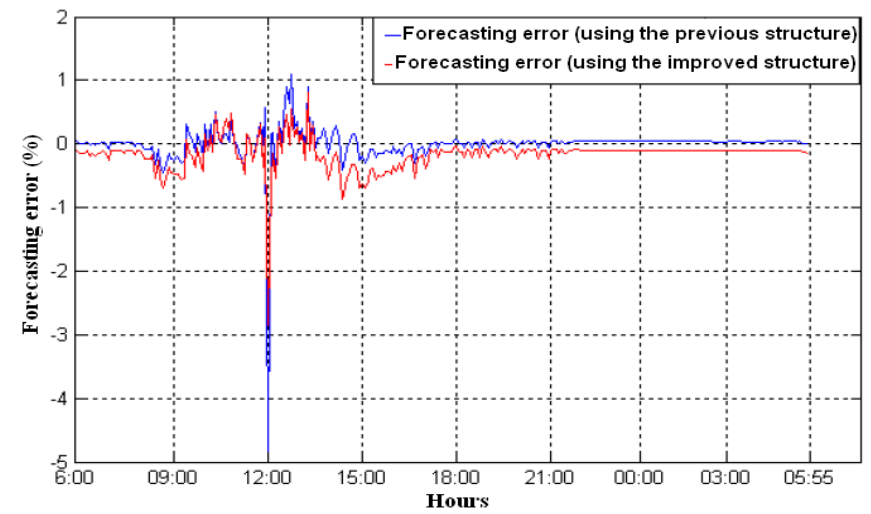

Fig.14. Forecasting error in the medium term using the improved structure

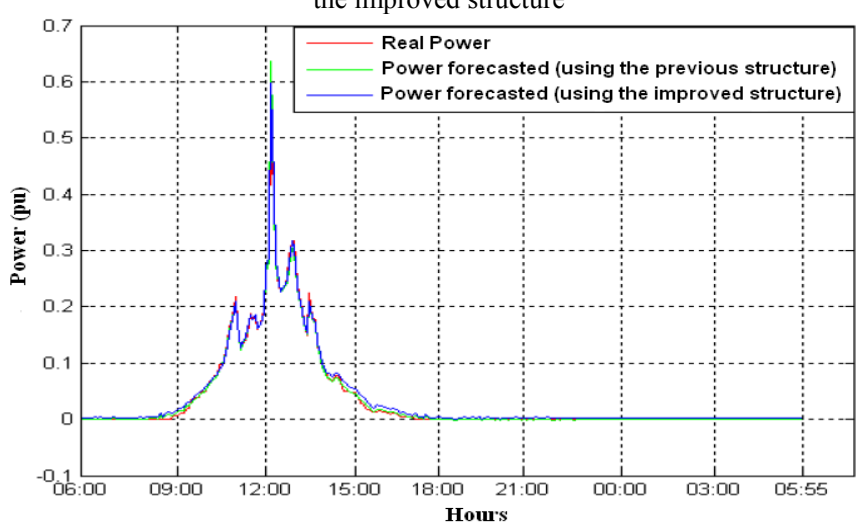

Fig.15. PV power forecasting on the medium term using the improved structure

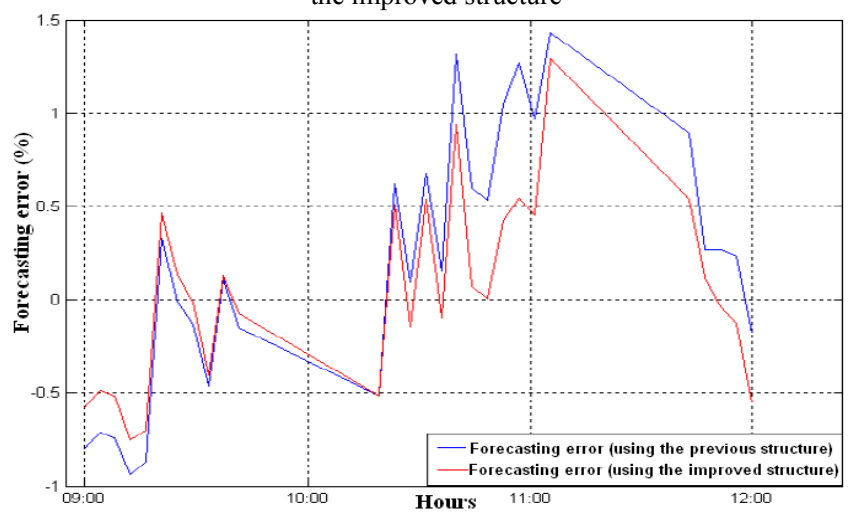

Fig.16. Forecasting error in the short term using the improved structure 


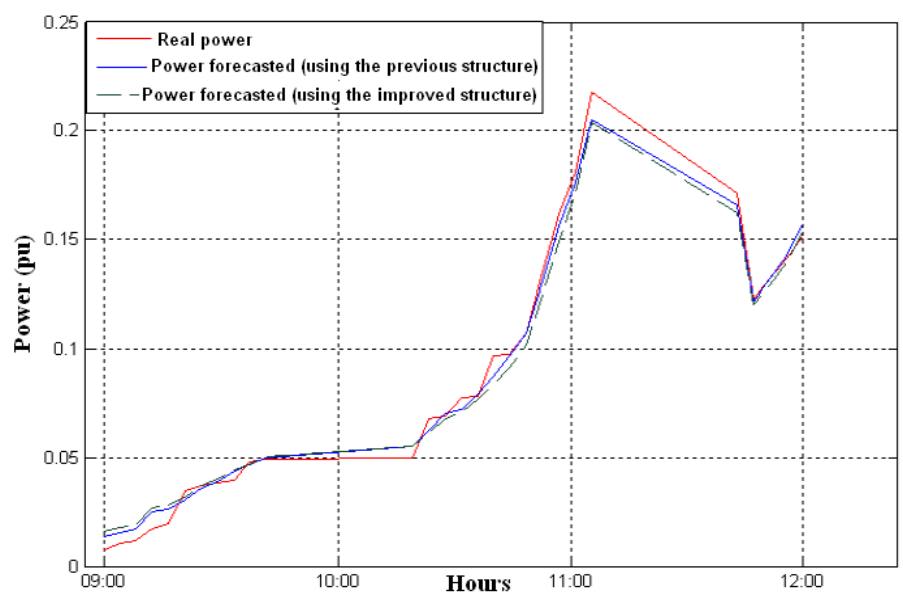

Fig.17. PV power forecasting on the short term using the improved structure

TABLE I. Forecasting error for the different horizon term

\begin{tabular}{|c|c|c|c|c|}
\hline \multirow{2}{*}{$\begin{array}{r}\text { ANN } \\
\text { strategies }\end{array}$} & $\begin{array}{c}\text { Model } \\
\text { identification }\end{array}$ & $\begin{array}{c}\text { Static } \\
\text { ANN }\end{array}$ & \multicolumn{2}{|c|}{ NARX } \\
\cline { 4 - 5 } Time frame & $\begin{array}{c}\text { Simple } \\
\text { structure }\end{array}$ & $\begin{array}{c}\text { Improved } \\
\text { structure }\end{array}$ \\
\hline $\begin{array}{c}\text { Long term } \\
\text { forecasting }\end{array}$ & (no computed) & 0.17 & 0.1 & 0.06 \\
\hline $\begin{array}{c}\text { Medium term } \\
\text { forecasting }\end{array}$ & 0.005 & 0.08 & 0.05 & 0.02 \\
\hline $\begin{array}{c}\text { Short term } \\
\text { forecasting }\end{array}$ & (no computed) & 0.027 & 0.017 & 0.012 \\
\hline
\end{tabular}

\section{CONCLUSION}

The present work represents a contribution of PV power forecasting using different ANN strategies. The results obtained were from a data base composed just of radiation, temperature and $\mathrm{PV}$ power correspond to a region located in the north of Barcelona. Firstly model identification is proposed to estimate some parameters of PV module and then the PV power is forecasted just in the medium term. Secondly static and a dynamic ANN are used to forecast the PV power from the radiation and the temperature. For short, medium and long time frame forecasting the performances of NARX model are verified. The effectiveness of NARX can be explained by its specificity to learn and generalize more effective than other types of ANN. In the last part of this paper the type of season is taking into account and it is introduced as a third input of NARX model. The forecasting results are improved especially for the short term.

However each data base probably contains redundant, missing and incorrect data so it is interesting to improve more these results by the treatment and the correction of this data base before forecasting.

\section{ACKNOWLEDGMENT}

"This work was supported by the Tunisian Ministry of High Education and Research under Grant LSE-ENIT-LR11ES1 and it was supported by the Tunisian Egyptian project".

\section{REFERENCES}

[1] Sant Joan les Fonts, Garrotxa, http://www.noel.es/

[2] Ahmed M. Yahya, I. Youm and A. Kader "Behavior and performance of a photovoltaic generator in real time, "International Journal of Physical Sciences, vol. 6(18), pp. 4361-4367, September 2011.

[3] Ernst.B, Reyer.F and Vanzetta.J "Wind power and photovoltaic prediction tools for balancing and grid operation," Integration of WideScale Renewable Resources Into the Power Delivery System, 2009 CIGRE/IEEE PES Joint Symposium, pp. 1-9, July 2009.

[4] Y.Huang, J.Lu, X.Xu, W.Wang and X.Zhou, "Comparative Study of Power Forecasting Methods for PV stations," International Conference on Power Technology, pp.1-6, Octobre 2010.

[5] Tao Cai, Shanxu Duan and Changsong Chen, "Forecasting power output for grid-connected photovoltaic power system without using solar radiation measurement,", 2nd IEEE International Symposium on Power Electronics for Distributed Generation Systems (PEDG), pp.773-777, June 2010.

[6] P. Bacher, H. Madsen and H. A. Nielsen, "Online short-term solar power forecasting," Solar Energy, vol. 83, pp. 1772-1783, 2009.

[7] M. Kudo, A. Takeuchi, Y. Nozaki, H. Endo and J. Sumita, "Forecasting Electric Power Generation in a Photovoltaic Power System for Energy Network," Electrical Engineering in Japan, vol. 1, pp.16-23, No. 4, June 2009.

[8] Chowdhury.B.H and Rahman.S, "Is central station photovoltaic power dispatchable," IEEE Transactions on Energy Conversion, vol.3, no.4, pp.747-754, Dec. 1988.

[9] A. Moreno-Munoz, J. de la Rosa, R. Posadillo and V. Pallares, "Short term forecasting of solar radiation," IEEE International Symposium on Industrial Electronics, ISIE 2008, pp.1537-1541, June 30-July 22008

[10]T. Hiyama and K. Kitabayashi, "Neural Network Based Estimation of Maximum Power Generation from PV Module using Environmental Information," IEEE Trans. on Energy Conversion, vol. 12, No. 3, 1997.

[11]F. Almonacid, C. Rus, P. J. Perez and L. Hontoria, "Estimation of the energy of a PV generator using artificial neural network," Renewable Energy, vol. 34, pp. 2743-2750, 2009.

[12] Yona, A.Senjyu, T.Saber, A.Y.Funabashi, T.Sekine and H. Chul-Hwan Kim, "Application of Neural Network to One-Day-Ahead 24 hours Generating Power Forecasting for Photovoltaic System," International Conference on Intelligent Systems Applications to Power Systems, ISAP 2007, pp.1-6, 5-8, Nov. 2007.

[13] A Azadeh, A.Maghsoudi and S.Sohrabkhani "An integrated artificial neural network approach for predicting global radiation," Energy conversion and management, vol.50, pp. 1497-1505, June 2009.

[14] Naji Yuehui HUANG, Jing LU, Chun LIU, Xiaoyan XU, Weisheng WANG and Xiaoxin ZHOU "Comparative study of power forecasting methods for PV stations," International Conference on Power System Technology (POWERCON), pp.1-6, Oct 2010.

[15] Stephane Petibon "Nouvelles architectures distribuées de gestion et de conversion de l'énergie pour les applications photovoltaïques" Université Paul Sabatier - Toulouse III (20/01/2009), C.ALONSO (Dir.). 